\title{
ARITHMETIC CHARACTERIZATIONS OF SIDON SETS
}

\author{
GILLES PISIER
}

\begin{abstract}
Let $\hat{G}$ be any discrete Abelian group. We give several arithmetic characterizations of Sidon sets in $\hat{G}$. In particular, we show that a set $\Lambda$ is a Sidon set iff there is a number $\delta>0$ such that any finite subset $A$ of $\Lambda$ contains a subset $B \subset A$ with $|B| \geq \delta|A|$ which is quasiindependent, i.e. such that the only relation of the form $\sum_{\lambda \in B} \epsilon_{\lambda} \lambda=0$, with $\epsilon_{\lambda}$ equal to \pm 1 or 0 , is the trivial one.
\end{abstract}

Let $G$ be a compact Abelian group and let $\hat{G}$ be the dual group. For any $f$ in $L_{2}(G)$, we denote by $\hat{f}$ the Fourier transform of $f$. A subset $\Lambda$ of $\hat{G}$ is called a Sidon set if there is a constant $K$ with the following property: all the trigonometric polynomials $f$, such that $\hat{f}$ is supported by $\Lambda$, satisfy

$$
\sum|\hat{f}(\gamma)| \leq K\|f\|_{C(G)} \text {. }
$$

We will denote by $S(\Lambda)$ the smallest constant $K$ with this property. In the theory of Sidon sets (cf. e.g. [2]), there has always been considerable interest in the relations between this analytical definition and the arithmetic properties of the set $\Lambda$ (in particular, in the case $G=\mathbf{T}$ and $\Lambda \subset \mathbf{Z}$ ). The aim of this note is to announce several arithmetic characterizations of Sidon sets.

Let us make more precise what we mean here by "arithmetic". We will denote by $R_{\Lambda}$ the set of relations (with coefficients in $\{-1,0,1\}$ ) satisfied by $\Lambda$, i.e. the set of all finitely supported families $\left(\epsilon_{\lambda}\right)_{\lambda \in \Lambda}$ in $\{-1,0,1\}^{\Lambda}$ such that $\sum_{\lambda \in \Lambda} \epsilon_{\lambda} \lambda=0$.

By an "arithmetic" characterization is usually meant one which depends only on the set $R_{\Lambda}$. In [1], Drury ${ }^{1}$ proved that such a characterization exists, but he could not produce any explicit one. Precisely, he proved the following: let $\Lambda$ and $\Lambda^{\prime}$ be two sets for which there is a bijection $\phi: \Lambda^{\prime} \rightarrow \Lambda$ such that the map $\tilde{\phi}: R_{\Lambda} \rightarrow R_{\Lambda^{\prime}}$, defined by $\tilde{\phi}\left(\left(\epsilon_{\lambda}\right)_{\lambda \in \Lambda}\right)=\left(\epsilon_{\phi\left(\lambda^{\prime}\right)}\right)_{\lambda^{\prime} \in \Lambda^{\prime}}$, is also a bijection. Then, $\Lambda$ is a Sidon set iff the same is true for $\Lambda^{\prime}$. In other words, the property of "being a Sidon set" is determined by $R_{\Lambda}$. We give below several explicit arithmetic characterizations, from which the preceding result of Drury follows as a corollary.

To state our results, we will need some notation and terminology. We will denote by $I_{\Lambda}$ the set of all finitely supported families $\left(\epsilon_{\lambda}\right)_{\lambda \in \Lambda}$ in $\{-1,0,1\}^{\Lambda}$. For any $\gamma$ in $\hat{G}$, we will denote by $R(\gamma, \Lambda)$ the number of ways to write $\gamma$ as

Received by the editors July 14, 1982.

1980 Mathematics Subject Classification. Primary 43A46, 42A55; Secondary 41A46, 41A65.

Key words and phrases. Sidon sets, dissociate sets, relations, arithmetic characterization.

${ }^{1}$ Drury considers only relations such that moreover $\sum_{\lambda \in \Lambda} \epsilon_{\lambda}=0$, but this difference is not significant, since we can replace $\Lambda$ by the set $\tilde{\Lambda} \subset \hat{G} \times \mathbf{Z}$ defined by $\tilde{\Lambda}=\{(\lambda, 1) \mid \lambda \in \Lambda\}$. 
a finite sum of the form $\gamma=\sum_{\lambda \in \Lambda} \epsilon_{\lambda} \lambda$ with $\left(\epsilon_{\lambda}\right)_{\lambda \in \Lambda}$ in $I_{\Lambda}$. For any integer $s \geq 0$, we will denote by $R_{s}(\gamma, \Lambda)$ the cardinal of the set of those $\left(\epsilon_{\lambda}\right)_{\lambda \in \Lambda}$ in $I_{\Lambda}$ such that $\sum\left|\epsilon_{\lambda}\right|=s$ and $\gamma=\sum_{\lambda \in \Lambda} \epsilon_{\lambda} \lambda$. (Note that we have obviously $R(\gamma, \Lambda)=\sum_{s>0} R_{s}(\gamma, \Lambda)$.) Let $A$ be a finite subset of $\Lambda$. We have the following identity, for all $\delta>0$.

$$
\prod_{\lambda \in A}[1+\delta(\lambda+\bar{\lambda})]=\sum_{\gamma \in \hat{G}} \gamma\left(\sum_{s \geq 0} \delta^{s} R_{s}(\gamma, A)\right) .
$$

We can now state our main theorem (we will denote by $|A|$ the cardinality of a set $A$ ).

THEOREM 1. Let $\Lambda$ be a subset of $\hat{G}$ not containing 0 . The following are equivalent.

(i) $\Lambda$ is a Sidon set.

(ii) There is a number $\theta<1$ such that, for all finite subsets $A$ of $\Lambda$, we have

$$
\sum_{s \geq 0} \frac{1}{2^{s}} R_{s}(0, A) \leq 2^{\theta|A|} .
$$

(iii) There is a number $\theta<1$ such that, for all finite subsets $A$ of $\Lambda$, we have

$$
\sup _{\gamma \in \hat{G}} R(\gamma, A) \leq 3^{\theta|A|} \text {. }
$$

(iv) There is a number $\theta<1$ such that, for all finite subsets $A$ of $\Lambda$, we have

$$
\left\{\sum_{\gamma \in \hat{G}} R(\gamma, A)^{2}\right\}^{1 / 2} \leq 3^{\theta|A|} .
$$

The details of the proof can be found in [5]. The equivalence (iii) $\Leftrightarrow$ (iv) is easy using the observation that $\sum_{G} R(\gamma, A)=3^{|A|}$. The proof of (i) $\Rightarrow$ (ii) uses (1) for $\delta=1 / 2$ and the integrability properties of $\sum_{\lambda \in A} \operatorname{Re} \lambda$. The proof relies very much on the previous paper [4] and on the following result which is proved in [5].

PROPOSITION. The conditions of Theorem 1 are also equivalent to the following.

(v) There are numbers $\alpha>0$ and $\rho<1$ such that, for any finite subset $A$ of $\Lambda$, we have

$$
m\left(\left\{t \in G \mid \inf _{\lambda \in A} \operatorname{Re} \lambda(t)>\rho\right\}\right) \leq 2^{-\alpha|A|} .
$$

(vi) There is a number $\alpha>0$ such that, for any finite subset $A$ of $\Lambda$, we can find points $t_{1}, \ldots, t_{N}$ in $G$, with $N \geq 2^{\alpha|A|}$ such that $\sup _{\lambda \in A}\left|\lambda\left(t_{i}\right)-\lambda\left(t_{j}\right)\right| \geq \alpha$ for all $i \neq j$.

The equivalence of (v) and (vi) is formal. The implication $(v) \Rightarrow(i)$ yields an affirmative answer to Problem 8.3 in [4].

DEFINITION. We will say that a set $\Lambda$ is a Rider set if there is some $\delta>0$ such that $\sum_{s \geq 0} \delta^{s} R_{s}(0, \Lambda)<\infty$. We will say that $\Lambda$ is quasi-independent if $R(0, \Lambda)=1$, or equivalently if $R_{s}(0, \Lambda)=0$ for all $s \geq 1$. 
Such sets-and finite unions of such sets-are the only known examples of Sidon sets, and the main open problem in this theory is the converse:

PROBLEM. Is every Sidon set a finite union of Rider sets? Is it a finite union of quasi-independent sets?

In the particular case $G=\mathbf{Z}(p)^{\mathbf{N}}$, with $p$ a prime number, a positive answer (as well as a complete arithmetic characterization) was given in [3]; very recently, J. Bourgain obtained a positive solution to the above problem, assuming more generally that $p$ is a product of distinct prime numbers (private communication).

Actually, it is rather easy to check (see [5]) that any Rider set is a finite union of quasi-independent sets; therefore, the above problem reduces to the second question.

Assume that a set $\Lambda$ is the union of $k$ quasi-independent sets. In that case, any finite subset $A$ of $\Lambda$, of cardinality $n$, must contain a quasi-independent subset $B \subset A$ with $|B| \geq n / k$. Therefore, if the above problem had a positive solution, any Sidon set should verify the above property for some $k$. It turns out that this is true.

THEOREM 2. A subset $\Lambda$ of $\hat{G}$ is a Sidon set iff

(vii) there is an integer $k$ such that any finite subset $A$ of $\Lambda$ contains a quasiindependent subset $B \subset A$ with $|B| \geq|A| / k$.

The proof that Sidon sets satisfy (vii) is given in [5]. The converse follows from Theorem 2.3 in [4], since any quasi-independent set $B$ is a Sidon set with $S(B)$ majorized by some absolute constant. In some sense, Theorem 2 reduces the above problem to a purely combinatorial question: Is every set satisfying (vii) a finite union of quasi-independent sets?

\section{REFERENCES}

1. S. Drury, Birelations and Sidon sets, Proc. Amer. Math. Soc. 53 (1975), 123-128.

2. J. López and K. Ross, Sidon sets, Lecture Notes in Pure and Appl. Math., no. 13, Dekker, New York, 1975.

3. M. P. Malliavin Brameret and P. Malliavin, Caractérisation arithmétique des ensembles de Helson, C. R. Acad. Sci. Paris Sér. A 264 (1967), 192-193.

4. G. Pisier, De nouvelles caractérisations des ensembles de Sidon, Advances in Math. Supplementary Studies, Mathematical Analysis and Applications, 7 B (1981), 685-726.

5. $\_$Condition d'entropie et caractérisations arithmétiques des ensembles de Sidon, Proc. Conf. on Modern Topics in Harmonic Analysis (Torino/Milano, June/July 1982), Instituto di Alta Mathematica (to appear).

Equipe D'ANAlyse, Université Paris Vi, 4, Place Jussieu, TOUR 46/0-4Éme ETAGE, 75230-PARIS CEDEX 05 